\title{
Corticosteroid treatment in severe COVID-19 patients with acute respiratory distress syndrome
}

\author{
Jiao Liu, ${ }^{1,2}$ Sheng Zhang, ${ }^{1}$ Xuan Dong, ${ }^{3}$ Zhongyi Li, ${ }^{4}$ Qianghong Xu, ${ }^{5}$ Huibin Feng, ${ }^{6}$ Jing Cai, ${ }^{7}$ Sisi Huang, ${ }^{2}$ Jun Guo, ${ }^{8}$ Lidi Zhang, \\ Yizhu Chen, ${ }^{2}$ Wei Zhu, ${ }^{9}$ Hangxiang Du, ${ }^{1}$ Yongan Liu, ${ }^{1}$ Tao Wang, ${ }^{1}$ Limin Chen, ${ }^{1}$ Zhenliang Wen, ${ }^{2}$ Djillali Annane, ${ }^{10}$ \\ Jieming Qu, ${ }^{11}$ and Dechang Chen ${ }^{1,2}$ \\ 'Department of Critical Care Medicine, Ruijin Hospital, Shanghai Jiao Tong University School of Medicine, Shanghai, China. ²Department of Critical Care Medicine, Ruijin Hospital North, Shanghai Jiao Tong \\ University School of Medicine, Shanghai, China. ${ }^{3}$ Tuberculosis and Respiratory Department, Wuhan Jinyin-tan Hospital, Wuhan, China. ${ }^{4}$ Department of Critical Care Medicine, Wuhan No. 9 Hospital, Wuhan, \\ China. ${ }^{5}$ Department of Critical Care Medicine, Zhejiang Hospital, Hangzhou, China. ${ }^{6}$ Intensive Care Unit, Huangshi Central Hospital, Affiliated Hospital of Hubei Polytechnic University, Edong Healthcare \\ Group, Huangshi, China. 'Department of Critical Care Medicine, Second Affiliated Hospital of Zhejiang University Medical College, Hangzhou, China. ${ }^{8}$ Intensive Care Unit, Huazhong University of Science and \\ Technology Union Jiangbei Hospital, Wuhan, China. ${ }^{9}$ Intensive Care Unit, Tianyou Hospital Affiliated to Wuhan University of Science and Technology, Wuhan, China. ${ }^{10} \mathrm{FHU}$ SEPSIS (Saclay and Paris Seine Nord \\ Endeavour to PerSonalize Interventions for Sepsis), RHU RECORDS (Rapi'd rEcognition of CORticosteroiD resistant or sensitive Sepsis), Department of Intensive Care, Hôpital Raymond Poincaré (APHP), \\ Laboratory of Infection and Inflammation - U1173, School of Medicine Simone Veil, University Versailles Saint Quentin - University Paris Saclay, INSERM, Garches, France. "Department of Pulmonary and \\ Critical Care Medicine, Ruijin Hospital, Shanghai Jiao Tong University School of Medicine, Shanghai, China.
}

BACKGROUND. Corticosteroids are widely used in patients with COVID 19, although their benefit-to-risk ratio remains controversial.

METHODS. Patients with severe COVID-19-related acute respiratory distress syndrome (ARDS) were included from December 29, 2019 to March 16, 2020 in 5 tertiary Chinese hospitals. Cox proportional hazards and competing risks analyses were conducted to analyze the impact of corticosteroids on mortality and SARS-CoV-2 RNA clearance, respectively. We performed a propensity score (PS) matching analysis to control confounding factors.

RESULTS. Of 774 eligible patients, 409 patients received corticosteroids, with a median time from hospitalization to starting corticosteroids of 1.0 day (IQR 0.0-3.0 days). As compared with usual care, treatment with corticosteroids was associated with increased rate of myocardial (15.6\% vs. $10.4 \%, P=0.041)$ and liver injury $(18.3 \%$ vs. $9.9 \%, P=0.001)$, of shock $(22.0 \%$ vs. $12.6 \%, P<0.001$ ), of need for mechanical ventilation ( $38.1 \%$ vs. $19.5 \%, P<0.001$ ), and increased rate of 28 -day all-cause mortality (44.3\% vs. 31.0\%, $P<0.001)$. After PS matching, corticosteroid therapy was associated with 28 -day mortality (adjusted HR 1.46, 95\% Cl 1.01-2.13, $P=0.045$ ). High dose ( $>200 \mathrm{mg}$ ) and early initiation ( $\leq 3$ days from hospitalization) of corticosteroid therapy were associated with a higher 28-day mortality rate. Corticosteroid use was also associated with a delay in SARS-CoV-2 coronavirus RNA clearance in the competing risk analysis (subhazard ratio 1.59, 95\% $\mathrm{Cl} 1.17-2.15, P=0.003$ ).

CONCLUSION. Administration of corticosteroids in severe COVID-19-related ARDS is associated with increased 28-day mortality and delayed SARS-CoV-2 coronavirus RNA clearance after adjustment for time-varying confounders.

FUNDING. None.

\section{Introduction}

SARS-CoV-2 has spread worldwide (1). As of July 17, there were more than 13 million cases and 585,727 deaths worldwide, including over 4000 reported deaths in China (1). There is still no specific treatment for COVID-19, and patient management relies on supportive care (2).

\section{Related Commentary: p. 6218}

Authorship note: JL, SZ, XD, ZL, QX, and HF contributed equally to this work. Conflict of interest: The authors have declared that no conflict of interest exists. Copyright: ( 2020, American Society for Clinical Investigation.

Submitted: May 26, 2020; Accepted: August 13, 2020; Published: November 3, 2020. Reference information: J Clin Invest. 2020;130(12):6417-6428.

https://doi.org/10.1172/JCl140617.
Approximately $31 \%-41.8 \%$ of hospitalized COVID-19 patients rapidly develop acute respiratory distress syndrome (ARDS), with subsequent increased risk of death $(3,4)$, and patient deterioration is likely related to dysregulated systemic inflammation (5), as highlighted by increases in serum levels of inflammatory cytokines (6). Corticosteroids downregulate systemic inflammation by nongenomic and genomic effects (7).

Corticosteroids have been widely used to treat severe pneumonia due to influenza A (H5N1) and SARS-CoV $(8,9)$. However, the benefits of corticosteroid treatment in patients with respiratory infection due to coronavirus has remained controversial. In a systematic review including 29 studies with corticosteroid therapy for SARS infection, 25 studies were inconclusive and 4 studies indicated harmful results (10). In a retrospective cohort study of 309 critically ill adults with Middle East respiratory syndrome 


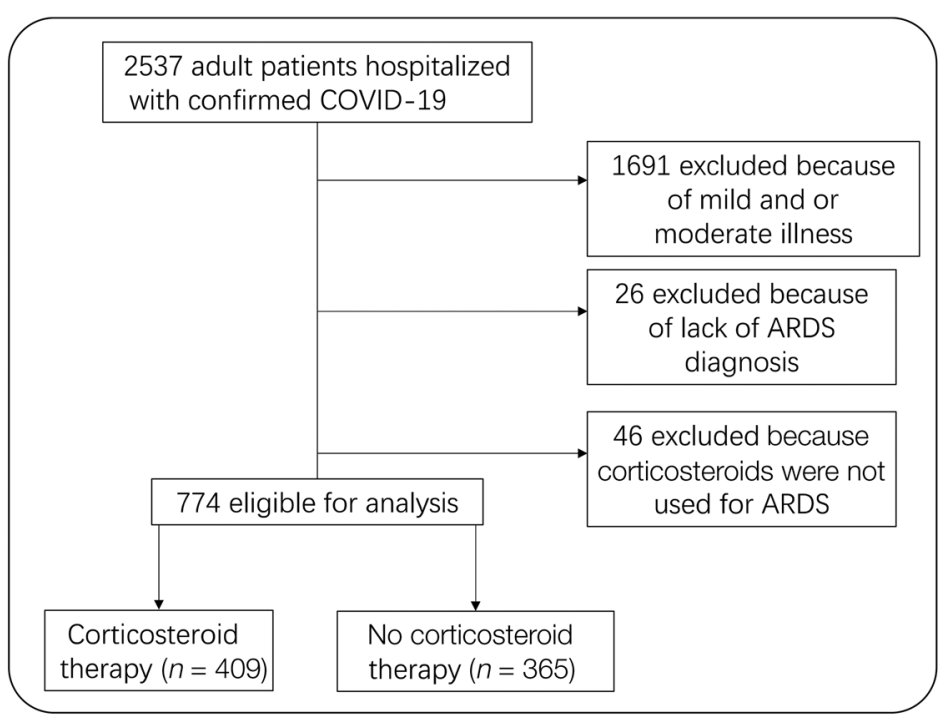

No new patients were hospitalized after March 16, 2020

Figure 1. Flow chart of the present study.

(MERS), corticosteroid therapy did not significantly change mortality and was associated with delayed viral clearance (11). An observational cohort of COVID-19 patients with ARDS suggested that treatment with methylprednisolone may decrease the risk of death (3). We conducted a retrospective multicenter study to explore the effects of corticosteroids on mortality from COVID-19 with ARDS. We also explored the effects of corticosteroids on the clearance of SARS-CoV-2 RNA. We aimed to explore the roles of corticosteroids and their influence on clinical outcomes in confirmed severe COVID-19-related ARDS.

\section{Results}

Description of the cohort. Of 2537 patients with confirmed COVID-19 admitted to 5 tertiary hospitals from December 29, 2019 to March 16, 2020, 820 had ARDS and 774 were eligible (Figure 1). The median age was 64 years (IQR 54-73 years) (Table 1) and 452 (58.0\%) were male, $322(42 \%)$ were female. A total of 596 patients $(77 \%)$ had one or more comorbidities. The median acute physiology and chronic health evaluation (APACHE) II score was 11 (IQR 9-13). A total of 409 out of 774 (52.8\%) received corticosteroids and 365 (47.2\%) did not. The median $\mathrm{PaO}_{2} / \mathrm{FiO}_{2}$ was 168 (IQR 99-237) before treatment in the whole cohort. On admission, 693 of 774 (89.5\%) patients received nasal cannula oxygen treatment, 43 of 774 (5.6\%) high-flow oxygen, 27 of 774 (3.5\%) noninvasive mechanical ventilation, and 11 of 774 (1.4\%) invasive mechanical ventilation. There was no significant difference in respiratory support between groups $(P=0.143)$. The crude 28-day all-cause mortality was $38.0 \%$ (294 of 774). Admission characteristics of ARDS patients are shown in Supplemental Table 1 (supplemental material available online with this article; https:// doi.org/10.1172/JCI140617DS1).

Corticosteroid therapy. Methylprednisolone was the most frequently used corticosteroid (396 of 409 [96.8\%] patients) followed by prednisolone (32 of 409 [7.8\%] patients) (Table 2). The median length from admission to initiation of corticosteroid ther- apy was 1.0 day (IQR 0.0-3.0 days) (Supplemental Figure 1), with a maximum daily hydrocortisone-equivalent median dose of $200 \mathrm{mg}$ (IQR 200-400 mg). The median duration of corticosteroid therapy was 6.0 days (IQR 4.0-10.0 days). Before corticosteroid administration, the median $\mathrm{PaO}_{2} / \mathrm{FiO}_{2}$ was $122 \mathrm{mmHg}$ (IQR 98-217 mmHg).

Mortality. At 28 days, there were 181 of 409 (44.3\%) deaths in the corticosteroid group and 113 of 365 (31.0\%) in the control group (OR 1.77, 95\% CI 1.32-2.38, $P<0.001$ ). A total of 185 out of 409 (45.2\%) corticosteroid-treated patients died during hospitalization, compared with 115 of 365 (31.5\%) controls (OR 1.79, 95\% CI 1.34-2.41, $P<0.001)$. The cumulative 28 -day survival rate was significantly different between the 2 groups $(P=0.00061$, log-rank test; Figure 2A). Multivariate logistic regression analysis suggested increased 28-day mortality with corticosteroids (adjusted OR 2.17, 95\% CI 1.36-3.53, $P=0.001$ ). Other independent risk factors for increased 28-day mortality included age, APACHE II score, diabetes, leukocytosis, and human immunoglobulin use (Table 3). Likewise, Cox's proportional hazards regression model also suggested increased mortality with corticosteroid therapy (adjusted HR 1.45, 95\% CI 1.06-1.99, $P=0.021$; Table 3). The association between corticosteroid use and mortality was more evident when treating corticosteroid as a time-varying exposure variable in the extended Cox regression model (adjusted HR 6.69, 95\% CI 4.53-9.87, $P<0.001$; Table 3). The increase risk of death at 28 days associated with exposure to corticosteroids was consistent across subgroups except for patients with APACHE II scores of 11 or higher who had shock or received mechanical ventilation (Figure 3). Among patients with shock, low-dose glucocorticoid therapy ( $\leq 200 \mathrm{mg}$ equivalent hydrocortisone per day) did not significantly decrease the risk of death (OR $0.41,95 \% \mathrm{CI}$ 0.12-1.33, $P=0.14$ ).

Finally, to determine the effect of corticosteroid therapy on 28-day mortality, we conducted a propensity score (PS) matching analysis. After applying PS matching, 182 control and 182 case patients were matched. The summaries of balance for unmatched and matched data are shown in Table 4 and Figure 4. Corticosteroid therapy was significantly associated with increased 28-day mortality in multiple analyses, including Kaplan-Meier survival plot (Figure 2B), multivariable logistic regression model (adjusted OR 1.64, 95\% CI 1.05-2.57, $P=$ 0.032; Table 5), and extended multivariable Cox regression model which treated corticosteroid as a time-varying exposure variable (adjusted HR 1.46, 95\% CI 1.01-2.13, $P=0.045$; Table $5)$. In subgroup analyses, high dose $(>200 \mathrm{mg})$ and early initiation ( $\leq 3$ days from hospitalization) of corticosteroid therapy were associated with a higher 28 -day mortality rate. In addition, even short course ( $\leq 6$ days) exposure to corticosteroids increased the risk of death (Table 5).

SARS-CoV-2 RNA clearance. Corticosteroid therapy was associated with delayed SARS-CoV-2 RNA clearance among survivors by log-rank test $(P=0.00017$; Figure $5 \mathrm{~A})$. Likewise in the whole cohort, the viral clearance was delayed with corticosteroid therapy (subhazard ratio [sHR] 1.59, 95\% CI 1.17-2.15, $P=0.003$; Figure $5 \mathrm{~B})$. The relationship between corticosteroid use and viral clear- 


\section{Table 1. Baseline characteristics and physiological parameters on admission of severe COVID-19 patients with ARDS in the corticosteroid-therapy and no-corticosteroid-therapy groups}

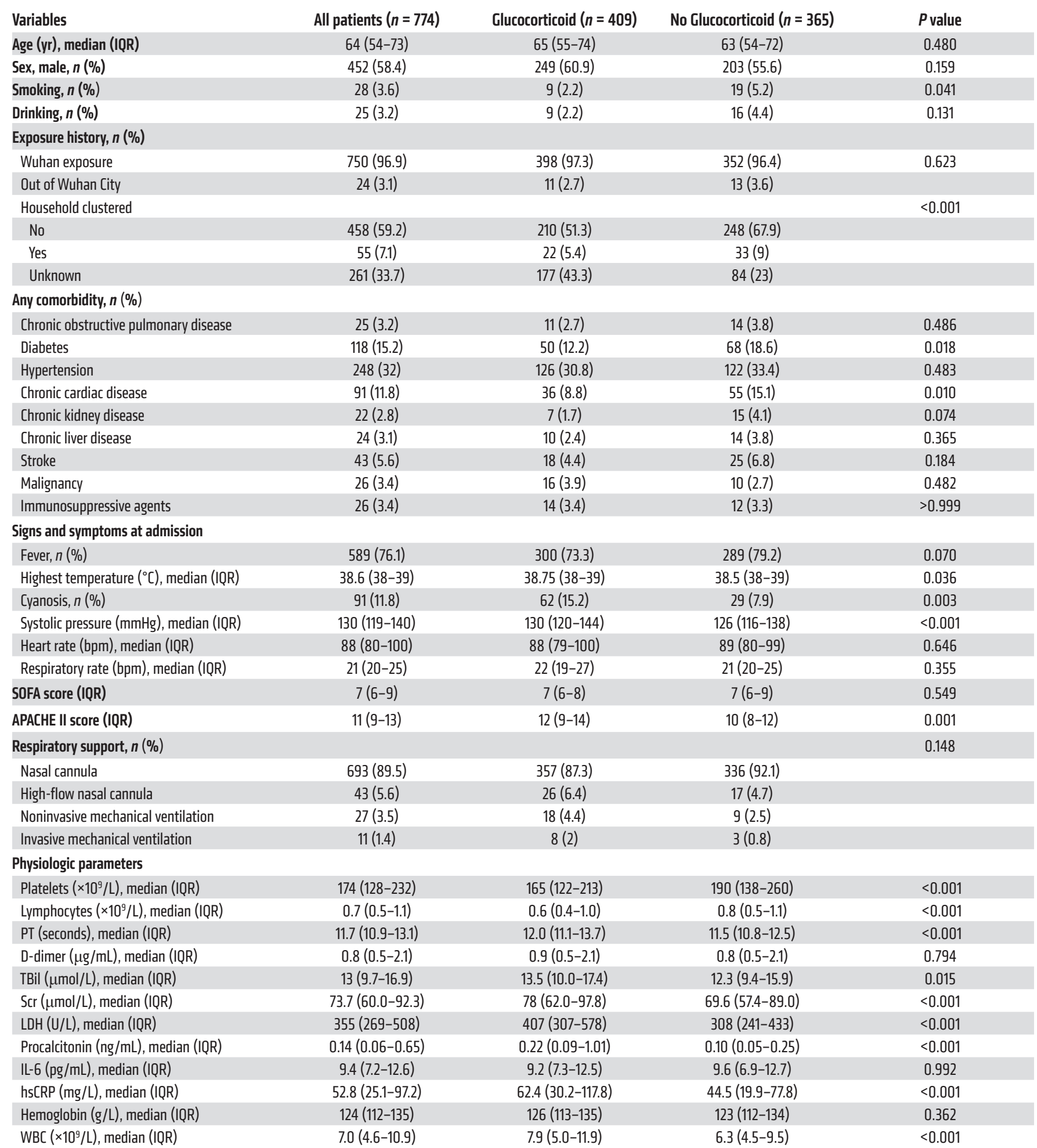

PT, partial thromboplastin time; TBil, total bilirubin; Scr, serum creatinine; LDH, lactate dehydrogenase; IL-6, interleukin-6; hsCRP, high sensitivity C-reactive protein. 
Table 2. Corticosteroid therapy among severe COVID-19 patients with ARDS $(n=409)$

\begin{tabular}{|c|c|}
\hline Medication variable & Result \\
\hline Dexamethasone, $n(\%)$ & $12(2.9)^{A}$ \\
\hline Hydrocortisone, $n(\%)$ & $2(0.5)^{A}$ \\
\hline Methylprednisolone, $n(\%)$ & $396(96.8)^{A}$ \\
\hline Prednisolone, $n(\%)$ & $32(7.8)^{A}$ \\
\hline \multicolumn{2}{|l|}{ Duration of corticosteroids, days (IQR) } \\
\hline All patients & $6(4$ to 10$)$ \\
\hline Survivors & $7(4$ to 11$)$ \\
\hline Nonsurvivors & 5 (3 to 9) \\
\hline \multicolumn{2}{|l|}{ Dose, hydrocortisone equivalent/day, mg (IQR) } \\
\hline All patients & $200(200$ to 400$)$ \\
\hline Survivors & $200(200$ to 400$)$ \\
\hline Nonsurvivors & $400(200$ to 400$)$ \\
\hline Duration between onset of illness and corticosteroid initiation, days (IQR) & 10 (7 to 14) \\
\hline Duration between hospital admission and corticosteroid initiation, days (IQR) & $1(0$ to 3$)$ \\
\hline Duration between ARDS onset and corticosteroid initiation, days (IQR) & $0(-1$ to 1$)$ \\
\hline Duration between ICU admission and corticosteroid initiation, days (IQR) & $0(-2$ to 0$)$ \\
\hline Duration between onset of non-invasive ventilation and corticosteroid initiation, days (IQR) & $0(-2$ to 0$)$ \\
\hline Duration between onset of invasive ventilation and corticosteroid initiation, days (IQR) & $-3(-8$ to -1$)$ \\
\hline
\end{tabular}

at 28 days and during hospitalization, and delayed SARS-CoV-2 clearance from the airway. The increase in mortality was consistent across subgroups with early initiation ( $\leq 3$ days from hospitalization), high dose (>200 mg), and independent of duration of corticosteroid treatment.

SARS-CoV-2-related ARDS is characterized by poor outcome, with $35 \%-40 \%$ of patients who have died in the short term (12). The mechanisms by which SARS-CoV-2 induces ARDS and eventually causes death remain uncertain. Similar to SARS-CoV infection, SARS-CoV-2 infection is associated with excessive lung and systemic inflammation (13) that contributes to the severity of illness (14) and the development of multiple organ failure (12). Corticosteroids are potent immunomodulatory drugs that downregulate inflammation by nongenomic and genomic effects (7). Therefore, they have been broadly used in adults with ARDS with variable results $(15,16)$. A metaanalysis from 4 trials suggested that in patients with SARS corticosteroids might have detrimental effects (10). Although ance did not change significantly in patients with early initiation of corticosteroid therapy (sHR 1.39, 95\% CI 1.02-1.89, $P=0.039$ ) and those with late initiation of corticosteroid therapy (sHR 2.79, 95\% CI 1.72-4.54, $P<0.001)$.

Other secondary outcomes. During hospitalization, as compared with controls, corticosteroid-treated patients were more likely to develop myocardial injury (15.6\% vs. $10.4 \%, P=0.041)$, acute liver injury (18.3\% vs. 9.9\%, $P=0.001)$, and shock $(22.0 \%$ vs. $12.6 \%, P<0.001)$. They were more likely to receive treatment with ganciclovir (29.3\% vs. $20.0 \%, P=0.004)$, ribavirin $(43.0 \%$ vs. $21.6 \%, P<0.001$ ), antibiotics (98.3\% vs. $76.7 \%, P<0.001)$, antifungal drugs $(10.5 \%$ vs. $1.1 \%, P<0.001)$, high-flow oxygen therapy $(33.5 \%$ vs. $16.2 \%, P<0.001)$, noninvasive mechanical ventilation $(25.9 \%$ vs. $14.0 \%, P<0.001)$, invasive mechanical ventilation ( $19.8 \%$ vs. $10.4 \%, P<0.001)$, and prone-position ventilation ( $4.6 \%$ vs. $1.4 \%, P=0.016)$ (Table 6 and Supplemental Table 2). As compared with controls, corticosteroid-treated patients were more likely to require ICU admission $(47.7 \%$ vs. $22.5 \%, P<0.001)$, had longer duration of stay in the hospital (median 15 days, IQR 9-21 days; compared with 13 days, IQR 8-18 days; $P<0.001$ ), and longer time in the ICU (median 5 days, IQR $1-11$ days; compared with 2 days, IQR 0-7 days; $P<0.001$ ) (Table 6). There was no significant difference between groups in the rate of occurrence of bacterial (5.8\% vs. $3.4 \%, P=0.336)$ and fungal $(8.0 \%$ vs. $8.3 \%, P>0.99)$ lower respiratory tract infections (Supplemental Table 3).

\section{Discussion}

This multicenter cohort study suggested that, in severe COVID-19related ARDS patients, corticosteroids increased the risk of death corticosteroids were not recommended during the 2003 SARS epidemic $(17,18)$, these drugs were broadly used by physicians. About half of critically ill adults with MERS received corticosteroids and were more likely to require mechanical ventilation, vasopressors, and renal replacement therapy during hospitalization (11). There was no evidence for any effect of corticosteroids on short-term mortality in these patients (11), independent of dose, duration, and initiation time.

During the COVID-19 pandemic, the indication for steroid use was mentioned in the New Coronavirus Pneumonia Diagnosis and Treatment Plan in China (http://www.cac.gov. cn/2020-03/04/c_1584872634644633.htm), which suggested low dose (1-2 mg/kg/d) and short duration (3-5 days) of methylprednisolone use in COVID-19 patients who had rapid $\mathrm{PaO}_{2} /$ $\mathrm{FiO}_{2}$-ratio and pulmonary-imaging deterioration after hospitalization. However, the decision to treat a patient with corticosteroid was left at the discretion of his/her primary physician. Fadel et al. found that hospitalized patients with moderate to severe COVID-19 who received an early short course of methylprednisolone had a reduced rate of mortality and mechanical ventilation (19). The RECOVERY trial, a platform open-label trial aimed at assessing multiple treatments for COVID-19 in hospitalized patients in the United Kingdom, found an age-adjusted rate ratio (aRR) of 28-day all-cause mortality of 0.83 (95\% CI 0.75-0.93) in favor of $6 \mathrm{mg}$ dexamethasone daily for 10 days (20). Nevertheless, in this trial, reduction in mortality with dexamethasone was seen only in patients who required supplemental oxygen with or without invasive mechanical ventilation. In patients who did not require supplemental oxygen, the aRR was 1.19 (95\% CI 0.911.55). A recent meta-analysis with a sample size of 5270 patients 
A
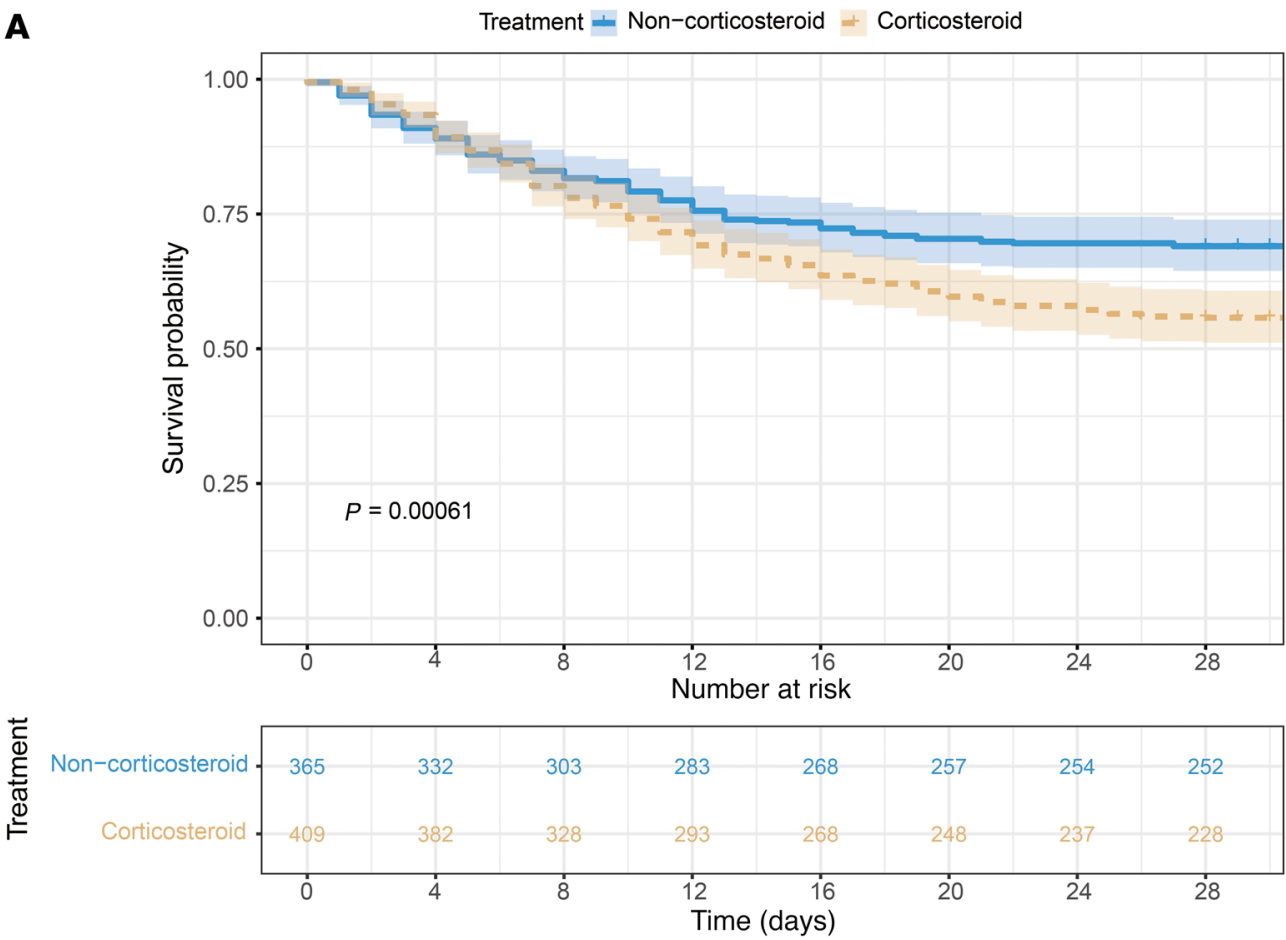

B
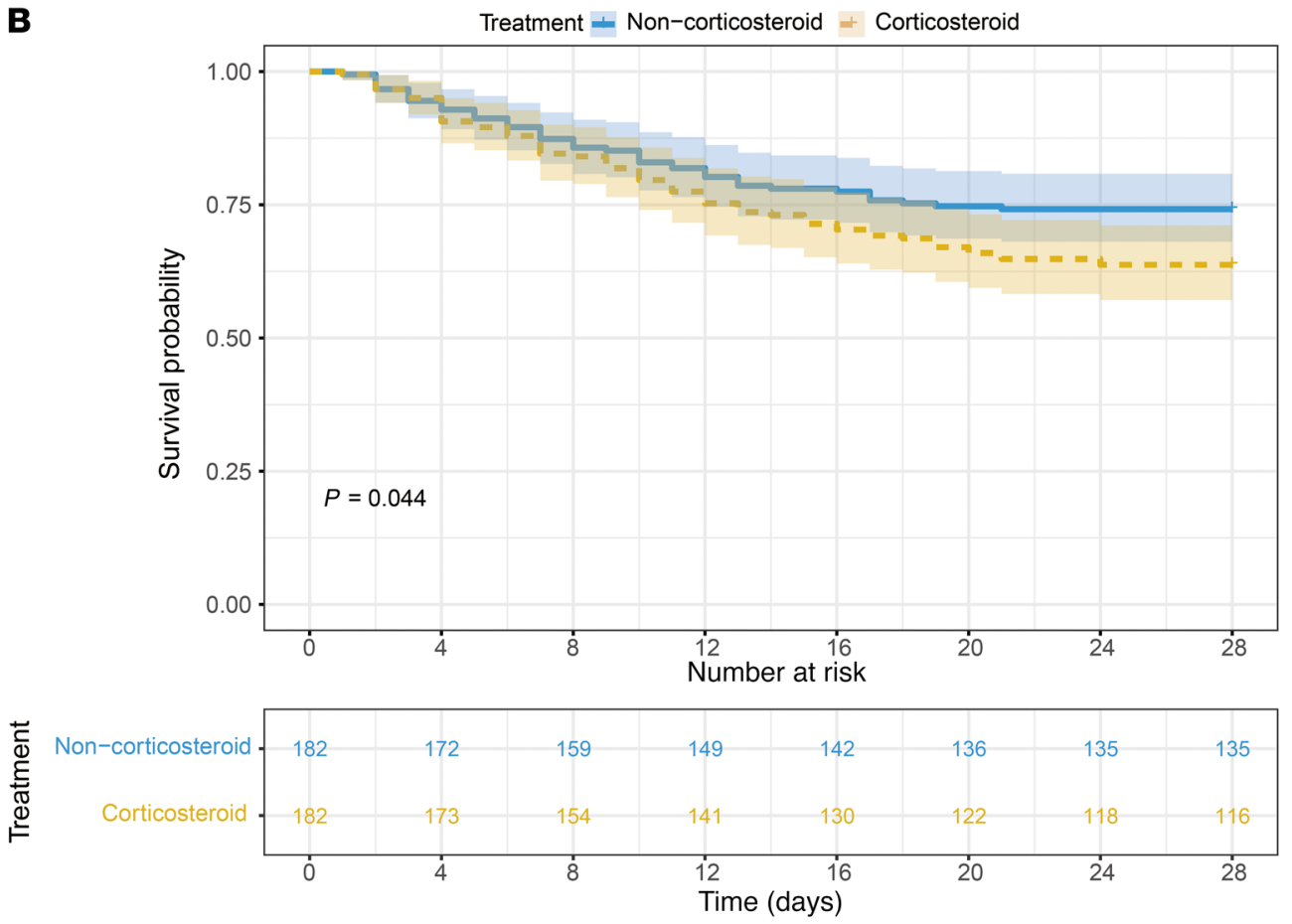

Figure 2. Survival curve during hospitalization according to corticosteroid therapy. Kaplan-Meier survival curves before (A) and after propensity score matching (B) suggested that treatment with corticosteroids was associated with increased mortality and prolonged hospital stays in patients with coronavirus pneumonia (21). The increase in mortality was consistent across subgroups with early initiation ( $\leq 3$ days from hospitalization) and high dose ( $>200 \mathrm{mg})$. The discrepancy in the findings from the current study and the RECOVERY trial may be related to differences in corticosteroid regimen and study populations. In the current study, most of the patients received methylprednisolone versus dexamethasone in the RECOVERY trial.
Pharmacological differences between methylprednisolone and dexamethasone include differences in mineralocorticoid activity (some versus none, respectively) and in plasma half-life (3 hours versus 72 hours, respectively). There were also differences in dose and duration of treatment with corticosteroids between the 2 studies with lower daily doses (150 versus 200 to $400 \mathrm{mg}$ of hydrocortisone equivalent, respectively) and longer duration (10 versus 6 days, respectively). Therefore, the inconsistent findings between the present study and previous studies $(19,20)$ may 
Table 3. Risk factors associated with 28-day mortality in severe COVID-19 patients with ARDS identified by logistic regression model, traditional Cox proportional hazards regression model, and extended Cox regression model

\begin{tabular}{|c|c|c|c|c|c|c|c|c|c|}
\hline \multirow[b]{2}{*}{ Variables } & \multicolumn{3}{|c|}{ Logistic regression model } & \multicolumn{3}{|c|}{ Traditional Cox model } & \multicolumn{3}{|c|}{ Extended Cox model ${ }^{\AA}$} \\
\hline & $\mathrm{aOR}$ & $95 \% \mathrm{Cl}$ & $P$ value & aHR & $95 \% \mathrm{Cl}$ & $P$ value & aHR & $95 \% \mathrm{Cl}$ & $P$ value \\
\hline Age & 1.04 & $1.03-1.06$ & $<0.001$ & 1.03 & $1.02-1.05$ & $<0.001$ & 1.04 & $1.02-1.05$ & $<0.001$ \\
\hline APACHE II score & 1.17 & $1.11-1.25$ & $<0.001$ & 1.11 & $1.08-1.15$ & $<0.001$ & 1.08 & $1.04-1.12$ & $<0.001$ \\
\hline COPD & 1.92 & $0.66-5.77$ & 0.233 & 1.50 & $0.77-2.90$ & 0.232 & 1.31 & $0.67-2.58$ & 0.432 \\
\hline Diabetes & 2.28 & $1.26-4.14$ & 0.007 & 1.58 & $1.12-2.25$ & 0.010 & 1.46 & $1.02-2.09$ & 0.040 \\
\hline Chronic kidney disease & 1.24 & $0.22-6.69$ & 0.803 & 1.3 & $0.47-3.58$ & 0.607 & 0.85 & $0.20-3.38$ & 0.832 \\
\hline Chronic liver disease & 0.49 & $0.13-1.59$ & 0.252 & 0.56 & $0.23-1.36$ & 0.203 & 0.62 & $0.23-1.65$ & 0.338 \\
\hline Stroke & 1.56 & $0.63-3.90$ & 0.334 & 0.99 & $0.58-1.70$ & 0.972 & 0.78 & $0.40-1.52$ & 0.461 \\
\hline Malignancy & 0.92 & $0.28-2.93$ & 0.890 & 1.14 & $0.54-2.41$ & 0.738 & 1.59 & $0.71-3.57$ & 0.259 \\
\hline Immunosuppression & 0.69 & $0.17-2.70$ & 0.596 & 0.52 & $0.21-1.25$ & 0.142 & 0.56 & $0.23-1.36$ & 0.198 \\
\hline Platelets & 1.00 & $0.99-1.00$ & 0.117 & 1.00 & $1.00-1.00$ & 0.034 & 1.00 & $1.00-1.00$ & 0.226 \\
\hline Lymphocytes & 0.91 & $0.66-1.24$ & 0.576 & 0.94 & $0.74-1.19$ & 0.616 & 1.01 & $0.79-1.29$ & 0.931 \\
\hline D-dimer & 1.02 & $1.00-1.04$ & 0.034 & 1.02 & $1.01-1.02$ & $<0.001$ & 1.02 & $1.01-1.03$ & $<0.001$ \\
\hline TBil & 1.03 & $1.00-1.06$ & 0.034 & 1.02 & $1.00-1.03$ & 0.025 & 1.02 & $1.01-1.04$ & 0.004 \\
\hline$s C r$ & 1.00 & $1.00-1.00$ & 0.679 & 1.00 & $1.00-1.00$ & 0.225 & 1.00 & $1.00-1.00$ & 0.615 \\
\hline Procalcitonin & 1.04 & $1.00-1.11$ & 0.161 & 1.01 & $1.00-1.03$ & 0.078 & 1.01 & $1.00-1.02$ & 0.210 \\
\hline Corticosteroids & 2.17 & $1.36-3.53$ & 0.001 & 1.45 & $1.06-1.99$ & 0.021 & 6.69 & $4.53-9.87$ & $<0.001$ \\
\hline Antiviral & 0.30 & $0.17-0.50$ & $<0.001$ & 0.43 & $0.32-0.60$ & $<0.001$ & 0.42 & $0.30-0.60$ & $<0.001$ \\
\hline Human immunoglobulin & 1.79 & $1.05-3.08$ & 0.035 & 1.55 & $1.09-2.22$ & 0.016 & 0.91 & $0.60-1.37$ & 0.643 \\
\hline
\end{tabular}

${ }^{A}$ Extended Cox model treating corticosteroid exposure as a time-varying exposure variable. COPD, chronic obstructive pulmonary disease; TBil, total bilirubin; sCr, serum creatinine; aHR, adjusted HR; aOR, adjusted OR.

be related to differences in the targeted population, mainly differences in severity and stage of the disease, as well as different practical modalities for corticosteroid treatment, i.e., different dose, timing, and duration. In the subset of patients with shock, the direction of the point estimate $(\mathrm{OR}=0.41)$ suggested potential benefits in this subset of patients with shock. However, the sample size was too limited, resulting in some imprecision in the result that precluded any definite conclusions. We also found that the use of corticosteroids in SARS-CoV-2-related ARDS was associated with higher prevalence of myocardial and hepatic injury, higher need for respiratory support, and subsequently increased risk of death.

Our findings of delayed SARS-CoV-2 RNA clearance in corticosteroid-treated patients are in line with previous reports in patients with SARS-CoV (22) and MERS-CoV (11) infections. The altered viral clearance may be related to corticosteroid effects on $\mathrm{T}$ cell responses and on interferon pathways (23). However, the persistence of the virus does not necessarily correlate with organ damage, as presence of SARS-CoV-2 RNA does not directly indicate shedding of live virus. A large retrospective cohort study demonstrated that viral RNA positivity persisted up to death, suggesting a possible correlation between viral persistence and poor prognosis (4).
To our knowledge, this is first large, multicenter observational study that addressed the effects of corticosteroids in severe COVID-19 patients with ARDS. The main strength of this study included a well-defined population, large size, and focus on corticosteroid therapy for ARDS and not for other reasons. Therefore, with a homogeneous group of patients with severe disease, and after careful minimization of confounding biases through a PS matching analysis and competing risks analysis, we offer strong evidence to support the association between corticosteroid therapy and increased mortality as well as delayed SARS-CoV-2 RNA clearance. However, there are still some limitations. The main limitations include the retrospective design and some heterogeneity in the population partly related to critically ill patients treated outside the ICU due to shortages in ICU beds. Some laboratory parameters (such as LDH, ferritin, and CRP) were missing for some patients on admission, which may have introduced some biases, although we did use multiple analytical strategies to increase the robustness of our estimates. In addition, the lack of a statistically significant increase in secondary infections related to corticosteroids may have resulted from insufficient power and should be taken with caution. We did not record practical modalities for lung-protective ventilation. Finally, there was no longterm follow-up. 


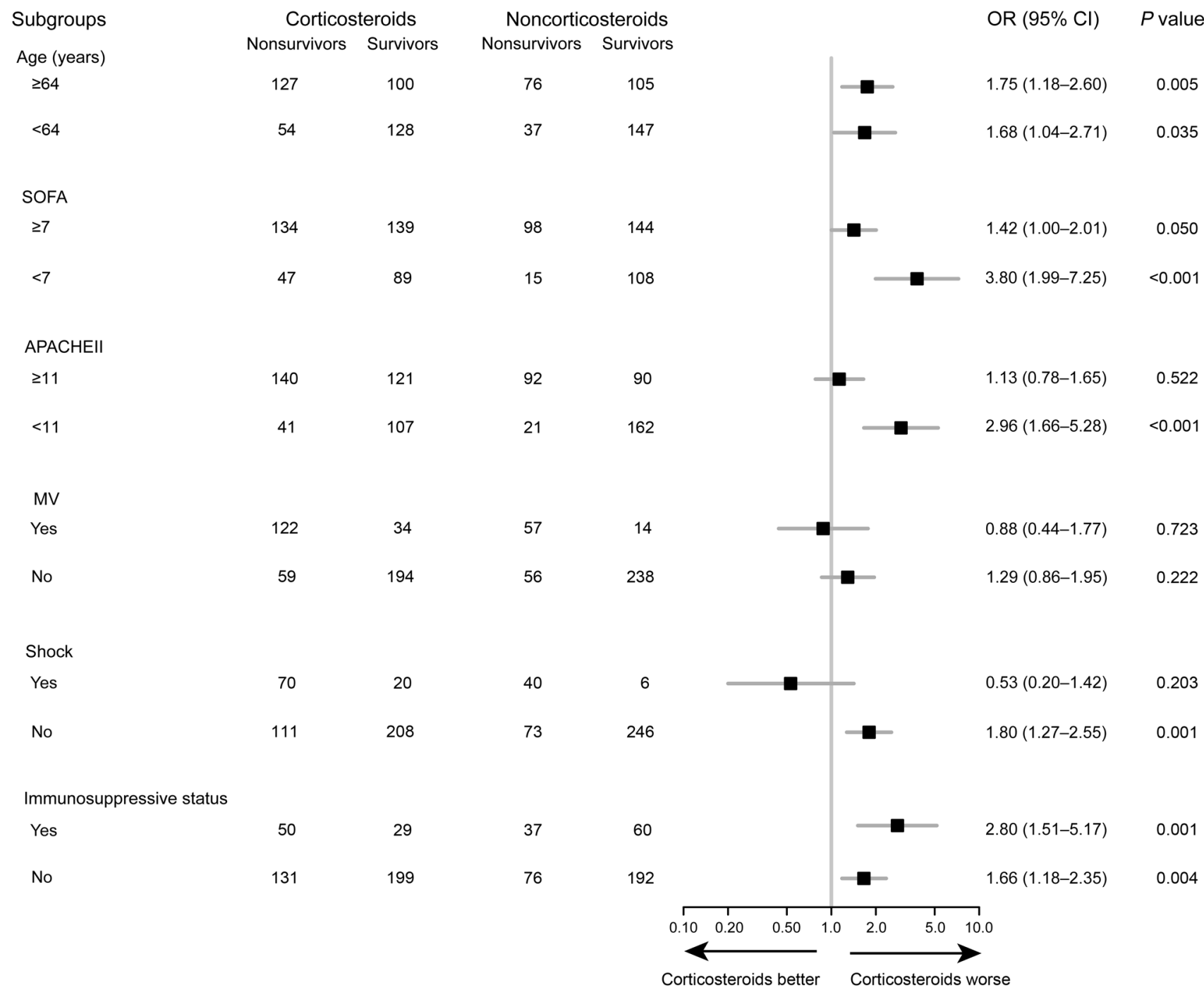

Figure 3. Subgroup analysis of 28-day mortality according to corticosteroid treatment in the whole cohort. APACHE II, acute physiology and chronic health evaluation II score; MV, mechanical ventilation; SOFA, sequential organ failure assessment.

\section{Methods}

\section{Study design}

This multicenter retrospective observational study was conducted in 5 tertiary hospitals (median hospital size, 578 beds [IQR 469-687]) from December 29, 2019 to March 16, 2020. The first part of the present study was to determine eligible patients who received corticosteroids and those who did not. To determine if the effect of corticosteroid therapy on 28-day mortality was different in subgroups, we examined the logistic regression analysis in the following subgroups: age $\geq 64$ years vs. age $<64$ years; APACHE II $\geq 11$ vs. APACHE II $<$ 11 ; with shock vs. without shock; sequential organ failure assessment (SOFA) $\geq 7$ vs. SOFA $<7$; with immunosuppressive status vs. without immunosuppressive status.

The second part of the study was to match patients who were exposed to corticosteroids at a 1:1 ratio with patients who were not exposed to corticosteroids. Matching was based on a multivariable logistic regression-generated PS for each patient without replacement.

\section{Study population}

We retrospectively collected medical records of adult patients with confirmed severe COVID-19-related ARDS who were admitted to 1 of the 5 participating tertiary hospitals, and who received corticosteroids for ARDS. We excluded patients who were already on long-term corticosteroid therapy and those who received corticosteroid therapy for other reasons, e.g., asthma, chronic obstructive pulmonary disease, etc.

\section{Intervention}

Patients with severe COVID-19-related ARDS were treated according to the Chinese national guidelines for the diagnosis and treatment of COVID-19. It was suggested that patients with severe COVID-19related ARDS should receive comprehensive treatments, including suitable respiratory support (lung-protective ventilation if necessary), antiviral therapy (lopinavir/ritonavir $100 \mathrm{mg}$, bid; Arbidol, $200 \mathrm{mg}$, tid, etc.), organ support, etc.

The intervention of interest for this cohort study was administration of corticosteroids for SARS-CoV-2-related ARDS, regardless 


\section{Table 4. Standard mean difference after propensity score matching}

Variables

\begin{tabular}{|c|c|c|c|}
\hline & No Glucocorticoid ( $n=182$ ) & Glucocorticoid $(n=182$ ) & SMD \\
\hline Age (yr), mean $( \pm S D)$ & $62.85 \pm 12.75$ & $63.42 \pm 14.35$ & 0.042 \\
\hline COPD, $n(\%)$ & $4(2.2)$ & $6(3.3)$ & 0.067 \\
\hline Diabetes, $n(\%)$ & $28(15.4)$ & $28(15.4)$ & $<0.001$ \\
\hline Hypertension, $n(\%)$ & $57(31.3)$ & $54(29.7)$ & 0.036 \\
\hline CCD, $n(\%)$ & $20(11.0)$ & $20(11.0)$ & $<0.001$ \\
\hline CKD, $n(\%)$ & $4(2.2)$ & $3(1.6)$ & 0.040 \\
\hline CLD, $n(\%)$ & $6(3.3)$ & $4(2.2)$ & 0.067 \\
\hline Stroke, $n(\%)$ & $7(3.8)$ & $8(4.4)$ & 0.028 \\
\hline Malignancy, $n(\%)$ & $5(2.7)$ & $8(4.4)$ & 0.089 \\
\hline Immunosuppression, $n(\%)$ & $6(3.3)$ & $6(3.3)$ & $<0.001$ \\
\hline Fever on admission, $n(\%)$ & $137(75.3)$ & $130(71.4)$ & 0.087 \\
\hline Respiratory support on admission, $n$ (\%) & & & 0.026 \\
\hline Nasal cannula, $n(\%)$ & $166(91.2)$ & $167(91.8)$ & \\
\hline High-flow nasal cannula, $n(\%)$ & $9(4.9)$ & $8(4.4)$ & \\
\hline Noninvasive mechanical ventilation, $n(\%)$ & $5(2.7)$ & $5(2.7)$ & \\
\hline Invasive mechanical ventilation, $n(\%)$ & $2(1.1)$ & $2(1.1)$ & \\
\hline Male sex, $n(\%)$ & $106(58.2)$ & $98(53.8)$ & 0.089 \\
\hline Systolic pressure on admission, $n(\%)$ & $129.49 \pm 20.15$ & $130.18 \pm 21.66$ & 0.033 \\
\hline Antiviral therapy, $n(\%)$ & $143(78.6)$ & $150(82.4)$ & 0.097 \\
\hline Immunoglobulin therapy, $n$ (\%) & $91(50.0)$ & $89(48.9)$ & 0.022 \\
\hline APACHE II score on admission, mean $( \pm S D)$ & $11.54 \pm 4.61$ & $11.45 \pm 3.76$ & 0.022 \\
\hline Leukocytes $\left(\times 10^{9} / \mathrm{L}\right)$, mean $( \pm \mathrm{SD})$ & $7.74 \pm 5.01$ & $7.91 \pm 3.78$ & 0.037 \\
\hline Hemoglobin (g/L), mean ( \pm SD) & $123.71 \pm 15.53$ & $122.87 \pm 16.80$ & 0.052 \\
\hline Platelets $\left(\times 10^{9} / \mathrm{L}\right)$, mean $( \pm \mathrm{SD})$ & $171.87 \pm 74.05$ & $177.81 \pm 59.50$ & 0.088 \\
\hline Lymphocytes $\left(\times 10^{9} / \mathrm{L}\right)$, mean $( \pm S \mathrm{D})$ & $0.87 \pm 0.62$ & $0.89 \pm 0.69$ & 0.037 \\
\hline D-dimer ( $\mu \mathrm{g} / \mathrm{mL})$, mean $( \pm \mathrm{SD})$ & $5.01 \pm 13.20$ & $4.56 \pm 12.86$ & 0.034 \\
\hline TBil $(\mu \mathrm{mol} / \mathrm{L})$, mean $( \pm \mathrm{SD})$ & $13.95 \pm 7.42$ & $13.64 \pm 5.57$ & 0.046 \\
\hline $\operatorname{Scr}(\mu \mathrm{mol} / \mathrm{L})$, mean $( \pm \mathrm{SD})$ & $86.24 \pm 81.94$ & $92.32 \pm 85.24$ & 0.073 \\
\hline Procalcitonin (ng/mL), mean ( $\pm \mathrm{SD})$ & $0.95 \pm 3.90$ & $0.93 \pm 2.32$ & 0.003 \\
\hline
\end{tabular}

SMD, standard mean difference; COPD, chronic obstructive pulmonary disease; CCD, chronic cardiac disease; CKD, chronic kidney disease; CLD, chronic liver disease; TBil, total bilirubin; Scr, serum creatinine. ness: (i) respiratory distress, respiratory rate $\geq 30$ per minute; (ii) oxygen saturation at room air at rest $\leq$ $93 \%$; or (iii) oxygen index less than $300 \mathrm{mmHg}$. We used the standard Berlin definition for ARDS (25-26), with noninvasive $\mathrm{SpO}_{2} / \mathrm{FiO}_{2}$ as a surrogate of $\mathrm{PaO} 2 / \mathrm{FiO}_{2}$, which was allowed for patients without $\mathrm{PaO}_{2}$ (27-29). An immunosuppressive status was defined as patients who had an underlying disease, such as human malignancy, liver cirrhosis, or chronic renal failure, diabetes, or receiving immunosuppressive therapy. Diagnosis of acute kidney injury (AKI) was according to the KDIGO clinical practice guidelines (30).

\section{Data collection}

We extracted the following from the medical records: (i) demographic data including age, sex, comorbidities, exposure history of coronavirus; (ii) clinical data: the course of SARSCoV-2 infection, blood-chemical test, blood-gas analysis; (iii) severity of illness based on APACHE II score (31) and SOFA score (32), presence of shock; (iv) corticosteroid use: type of drug, initial and maximum daily dosage (converted to equivalent hydrocortisone dose), duration of treatment, time from initiation of mechanical ventilation; and (v) respiratory support, including oxygen therapy, noninvasive mechanical ven-

of the type of molecule, the dose, and the duration of treatment. The dose of corticosteroids is expressed as the hydrocortisone equivalent (methylprednisolone 1:5, dexamethasone 1:25, prednisolone 1:4). The control intervention was usual care.

\section{Definitions}

COVID-19 diagnosis was made according to WHO interim guidance (22). Nasal and pharyngeal swab specimens from patients with history of epidemiology and characteristics of viral pneumonia in chest CT or x-ray were obtained. The time interval between 2 specimens was at least 24 hours. Detection of SARS-CoV-2 nucleic acid was performed as previously described (https://www.who.int/publications/i/ item/10665-331501). Patients with at least 2 consecutive positive results from high-throughput sequencing or real-time reverse transcriptase polymerase chain reaction (RT-PCR) assay of nasal and pharyngeal swab specimens were confirmed as having COVID-19.

Severe patients were defined according to the Diagnosis and treatment of COVID-19 guidelines (sixth version) published by the National Health Commission of China (24). Patients who met one of the conditions listed below were diagnosed as having severe ill- tilation, invasive mechanical ventilation, prone-position ventilation, and extracorporeal membrane oxygenation (ECMO).

\section{Endpoints}

The primary endpoint was 28-day all-cause mortality. The key secondary endpoint was length from symptom onset to SARS-CoV-2 RNA clearance in respiratory secretions. Other secondary outcomes included in-hospital mortality; length of ICU and hospital stay; ICU admission; development of ARDS, disseminated intravascular coagulation (DIC), myocardial injury, acute hepatic injury (AHI), shock, AKI, respiratory support, prone-position ventilation, continuous renal replacement therapy (CRRT), ECMO, and presence of secondary infection.

\section{Statistical analysis}

There was no formal computation of sample size. We compared baseline characteristics, interventions, and outcomes in patients who received corticosteroid therapy with those in patients who did not. Continuous variables are expressed as the mean ( \pm standard deviation), or as median (IQR 25\%-75\%), and compared using 2-tailed Student's $t$ test or Mann-Whitney $U$ test. Discrete variables are expressed 


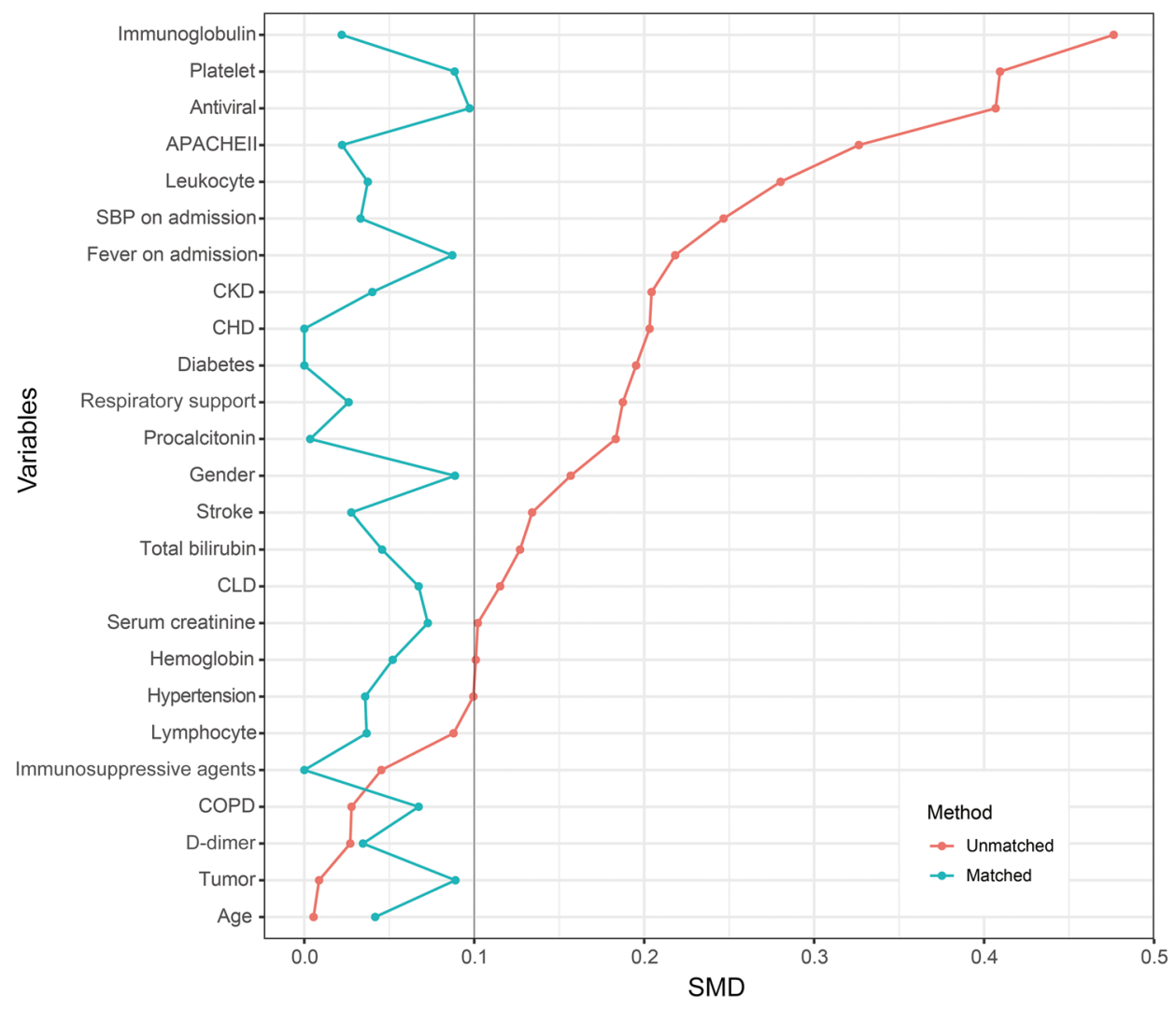

Figure 4. Summaries of the balance of variables before and after propensity score matching. SBP, systolic blood pressure; CKD, chronic kidney disease; CHD, chronic heart disease; CLD, chronic liver disease; COPD, chronic obstructive pulmonary disease; SMD, standard mean difference. as number (percentage), and compared using $\chi^{2}$ test or Fisher's exact test, as appropriate.

\section{Association of corticosteroid therapy and 28-day mortality}

Logistic regression analysis. The effects of corticosteroids on 28-day allcause mortality were explored by a multivariable logistic regression model. The model was adjusted on a priori-decided baseline variables of clinical interest and on those with a $P$ value of less than 0.05 in the univariate analysis. The included variables were age, sex, day 1 APACHE II score, COPD, chronic cardiac disease, chronic kidney disease, chronic liver disease, stroke, malignancy, diabetes with chronic complications, fever and systolic blood pressure at admission, leukocytes, platelets, lymphocytes, D-dimer, total bilirubin, creatinine, procalcitonin, therapy including immunoglobulin, and antiviral drugs. We chose respiratory support rather than $\mathrm{PaO}_{2} / \mathrm{FiO}_{2}$ to reflect respiratory status, as this variable was well documented for all patients. We excluded the variables SOFA score, diastolic blood pressure at admission, neutrophil count, prothrombin time, levels of C-reactive protein and $\mathrm{LDH}$, and ferritin, owing to colinearity or to an unacceptable rate of missing data.

The effects of corticosteroids therapy on 28-day all-cause mortality were also explored in the following subgroups: age $\geq 64$ years vs. age $<64$ years; APACHE II $\geq 11$ vs. APACHE II < 11; with shock vs. without shock; SOFA $\geq 7$ vs. SOFA $<7$; and with vs. without immunosuppressive status. The cutoff value for continuous variables in each subgroup was determined according to the median value of our population.

Cox's proportional hazards regression. To estimate the association between corticosteroid use and mortality as a time to event, we used the multivariable Cox regression model. An initial multi- variable Cox regression model incorporated corticosteroid therapy as a categorical variable with adjustment for the same above-mentioned variables used in the logistic regression model. In addition, to account for the time-varying exposure of corticosteroids, we fitted an extended Cox's regression model by considering the corticosteroids as a time-varying exposure variable with adjustment for the same above-mentioned variables.

\section{Association of corticosteroid therapy and SARS-CoV-2 RNA clearance} Kaplan-Meier plot and log-rank test. We used the Kaplan-Meier plot and log-rank test to analyze the time to SARS-CoV-2 RNA clearance. For this purpose, we only selected patients if they survived during hospitalization and had complete timelines of SARS-CoV-2 RNA clearance. We computed cumulative rates of SARS-CoV-2 RNA clearance over time for patients who received or did not receive corticosteroid therapy.

Competing risks analysis. Because a proportion of patients did not have SARS-CoV-2 RNA clearance until death, the Cox hazards model is not satisfactory for describing SARS-CoV-2 RNA clearance over time. We therefore performed a competing risk analysis using the Fine and Gray model, which considered events of interest (SARSCoV-2 RNA clearance) and competing events (death) in the same model. In addition, we explored whether early initiation ( $\leq 3$ days of hospitalization) or late initiation ( $>3$ days of hospitalization) of corticosteroid therapy would affect SARS-CoV-2 RNA clearance. The association between each variable and the outcome was estimated by the sHR with $95 \%$ CI.

In both the Kaplan-Meier survival plot and competing risks analysis, we only included patients who initiated corticosteroid therapy before SARS-CoV-2 RNA clearance to ensure a causal relationship. 
A
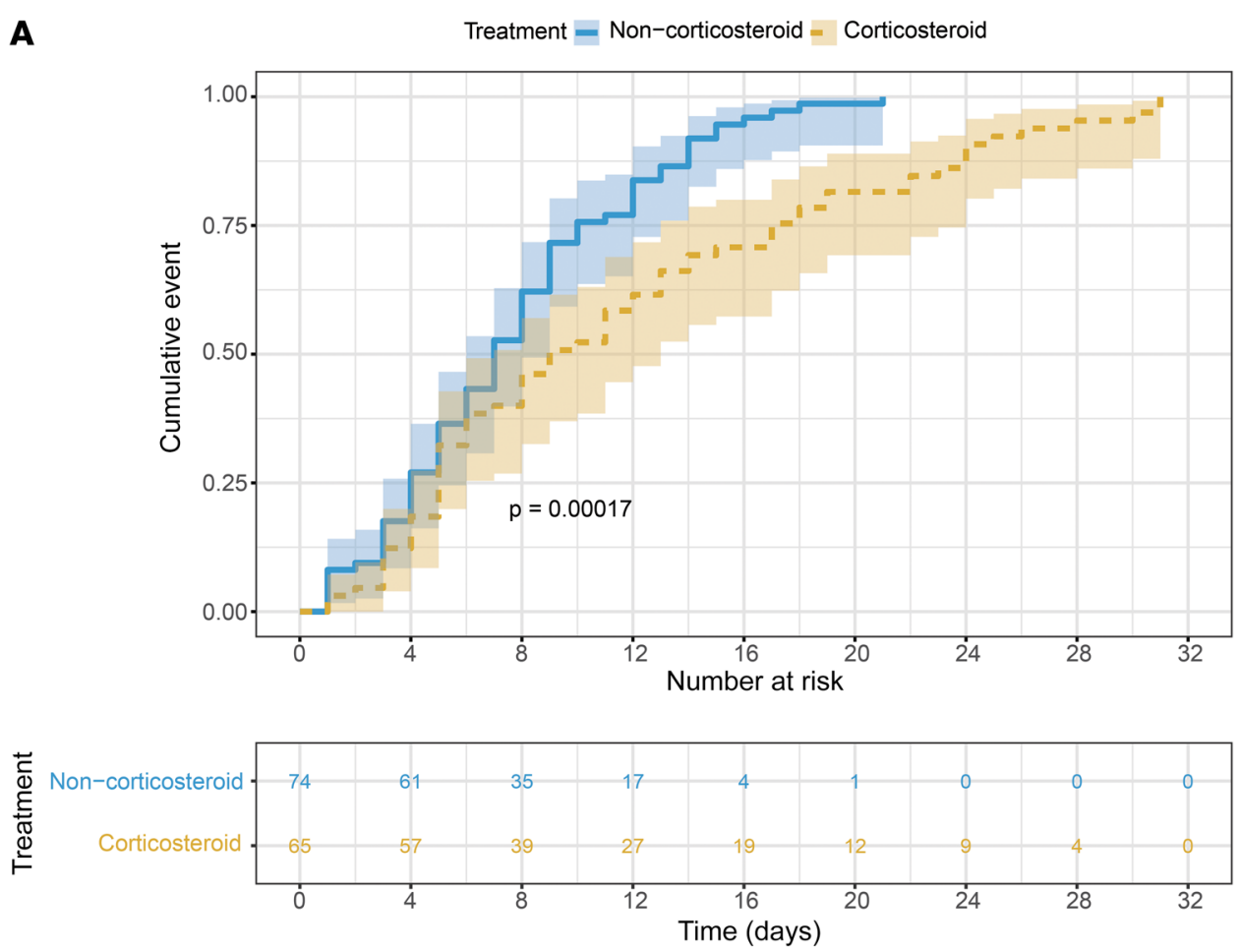

B

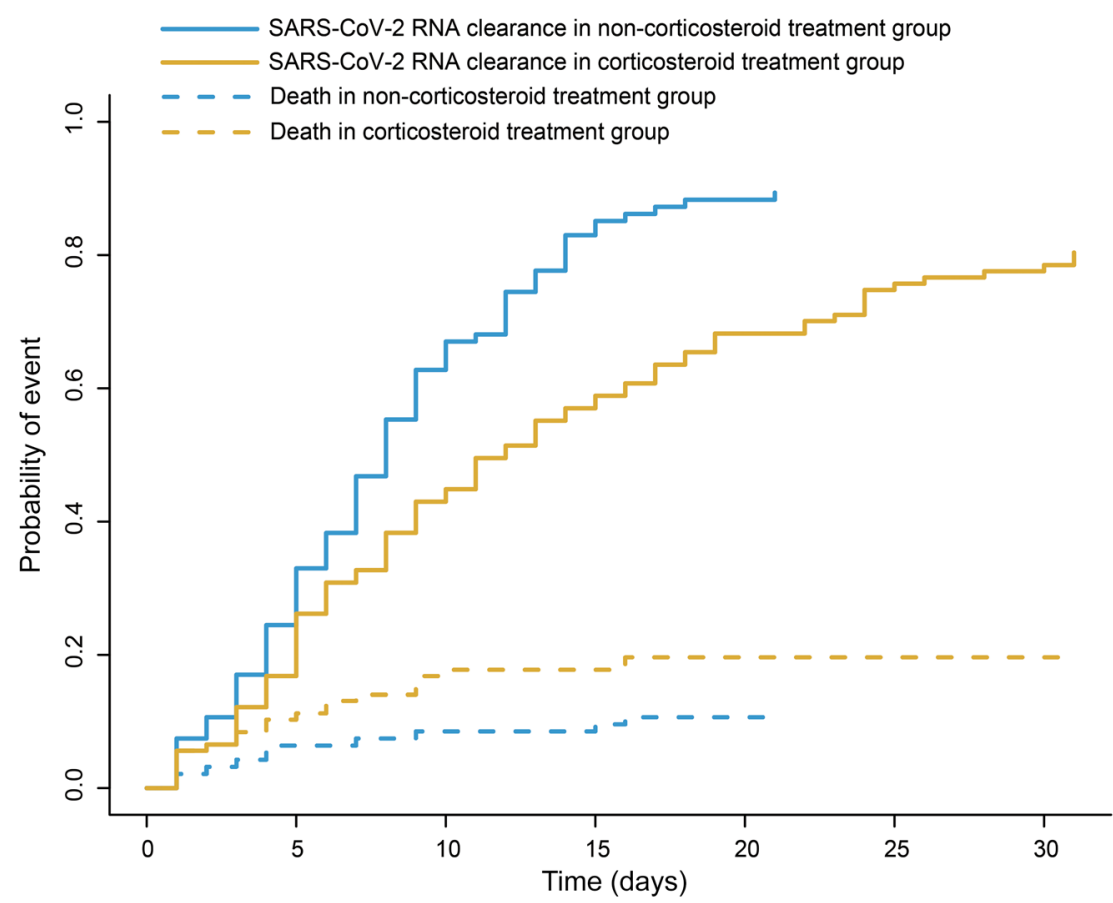

Figure 5. Cumulative incidence of SARS-CoV-2 RNA clearance according to corticosteroid therapy. (A) Cumulative incidence of SARS-CoV-2 RNA clearance according to corticosteroid therapy by Kaplan-Meier plot. (B) Cumulative incidence of SARS-CoV-2 RNA clearance and 28-day mortality by competing risks analysis.
Full-matching PS analysis. We performed a one-to-one PS matching analysis to account for potential confounding factors. This would allow us to compare outcomes between 2 cohorts of patients who had similar baseline characteristics except for treatment variable (receiving or not receiving corticosteroid therapy). PS was calculated for each patient based on a logistic-regression model that included the same variables used in the above-mentioned logistic regression model and Cox regression model. Matching was based on the logit of the PS using nearest-neighbor matching (greedy-type matching) with a caliper width of 0.2. Standardized mean differences for all covariates before and after matching were estimated and a difference of $10 \%$ or greater was considered to be indicative of imbalance. After matching, Kaplan-Meier curves were used to track the 28-day mortality for patients receiving or not receiving corticosteroid therapy. In addition, an extended Cox regression model that treated corticosteroids as a time-varying exposure variable was used to assess the effect of corticosteroid therapy on 28-day mortality.

Subgroup analysis after PS matching. To examine whether the effects of corticosteroid therapy on 28-day all-cause mortality were modified by varied dose, duration, and timing of initiation, we 


\section{Table 5. Twenty-eight-day mortality of severe COVID-19 patients with ARDS using various adjustment methodologies (after propensity score matching)}

Variables

All patients treated with corticosteroids vs. not treated with corticosteroids

Patients treated with corticosteroids $>200 \mathrm{mg}$ vs. not treated with corticosteroids

Patients treated with corticosteroids $\leq 200 \mathrm{mg}$ vs. not treated with corticosteroids

Patients treated with corticosteroids $\leq 6$ days vs. not treated with corticosteroids

Logistic regression model

$\begin{array}{ccccccc}n & \text { OR } & \text { 95\% Cl } & \boldsymbol{P} \text { value } & \text { HR } & \text { 95\% Cl } & \boldsymbol{P} \text { value } \\ 182 \text { vs. } 182 & 1.64 & 1.05-2.57 & 0.032 & 1.46 & 1.01-2.13 & 0.045 \\ 97 \text { vs. } 182 & 2.13 & 1.26-3.60 & 0.005 & 1.76 & 1.15-2.67 & 0.008 \\ 85 \text { vs. } 182 & 1.16 & 0.65-2.06 & 0.611 & 1.13 & 0.69-1.84 & 0.623 \\ 102 \text { vs. } 182 & 1.77 & 1.05-2.98 & 0.031 & 1.64 & 1.07-2.51 & 0.022 \\ 80 \text { vs. } 163 & 2.54 & 1.36-4.76 & 0.003 & 2.14 & 1.26-3.64 & 0.005 \\ 38 \text { vs. } 182 & 1.67 & 0.78-3.47 & 0.174 & 1.40 & 0.77-2.54 & 0.272 \\ 144 \text { vs. } 182 & 1.63 & 1.01-2.63 & 0.045 & 1.48 & 1.00-2.19 & 0.050\end{array}$

Corticosteroid initiation $\leq 3$ days vs. not treated with corticosteroids

variable. $\mathrm{OR}$, odds ratio; $\mathrm{HR}$, hazard ratio; $\mathrm{Cl}$, confidence interval.

\section{Table 6. Clinical course and outcomes among severe COVID-19 patients with ARDS in the corticosteroid-therapy and no-corticosteroid- therapy groups}

\begin{tabular}{|c|c|c|c|}
\hline Medication Variable & Glucocorticoid $(n=409)$ & No Glucocorticoid $(n=365)$ & $P$ value \\
\hline In-hospital mortality, $n(\%)$ & $185(45.2)$ & $115(31.5)$ & $<0.001$ \\
\hline 28-day mortality, $n(\%)$ & $181(44.3)$ & $113(31.0)$ & $<0.001$ \\
\hline Length of hospitalization (days), median (IQR) & $15(9-21)$ & $13(8-18)$ & $<0.001$ \\
\hline Length of ICU stay (days), median (IQR) & $5(1-11)$ & $2(0-7)$ & $<0.001$ \\
\hline Duration between COVID-19 onset and hospital admission (days), median (IQR) & $9.0(6.0-12.0)$ & $9.0(6.0-14.0)$ & 0.054 \\
\hline Duration between COVID-19 onset and ICU admission (days), median (IQR) & $11.0(7.0-15.0)$ & $12.0(8.0-16.0)$ & 0.569 \\
\hline ICU admission, $n(\%)$ & $195(47.7)$ & $82(22.5)$ & $<0.001$ \\
\hline Duration between COVID-19 onset and ARDS onset (days), median (IQR) & $11.0(7.0-15.0)$ & $10.0(3.0-15.0)$ & 0.160 \\
\hline ARDS, $n(\%)$ & $409(100)$ & $365(100)$ & 1.000 \\
\hline Duration between COVID-19 onset and DIC onset (days), median (IQR) & $18.0(14.0-23.0)$ & $19.0(14.0-20.5)$ & 0.781 \\
\hline DIC, $n(\%)$ & $13(3.2)$ & $7(1.9)$ & 0.381 \\
\hline Duration between COVID-19 onset and myocardial injury onset (days), median (IQR) & $16.0(11.0-21.0)$ & $17.0(10.3-23.0)$ & 0.970 \\
\hline Myocardial injury, $n(\%)$ & $64(15.6)$ & $38(10.4)$ & 0.041 \\
\hline Duration between COVID-19 onset and AHI onset (days), median (IQR) & $15.0(10.0-21.0)$ & $15.5(11.0-21.0)$ & 0.975 \\
\hline $\mathrm{AHI}, n(\%)$ & $75(18.3)$ & $36(9.9)$ & 0.001 \\
\hline Duration between COVID-19 onset and shock onset (days), median (IQR) & $17.0(13.0-22.8)$ & $18.0(13.0-22.8)$ & 0.934 \\
\hline Shock, $n(\%)$ & $90(22.0)$ & $46(12.6)$ & $<0.001$ \\
\hline Duration between COVID-19 onset and AKI (days), median (IQR) & $19.0(13.0-23.0)$ & $15.5(11.0-20.3)$ & 0.148 \\
\hline AKI, $n(\%)$ & $41(10.0)$ & $30(8.2)$ & 0.457 \\
\hline Duration between COVID-19 onset and high-flow oxygen therapy (days), median (IQR) & $12.0(8.0-17.0)$ & $12.0(9.0-16.5)$ & $>0.99$ \\
\hline High-flow oxygen therapy, $n(\%)$ & $137(33.5)$ & $59(16.2)$ & $<0.001$ \\
\hline Duration between COVID-19 onset and noninvasive mechanical ventilation (days), median (IQR) & $12.0(9.0-16.8)$ & $13.0(8.5-19.5)$ & 0.632 \\
\hline Noninvasive mechanical ventilation, $n(\%)$ & $106(25.9)$ & $51(14.0)$ & $<0.001$ \\
\hline Duration between COVID-19 onset and invasive mechanical ventilation (days), median (IQR) & $15.0(11.0-20.0)$ & $16.5(11.0-23.5)$ & 0.410 \\
\hline Invasive mechanical ventilation, $n$ (\%) & $81(19.8)$ & $38(10.4)$ & $<0.001$ \\
\hline Duration between COVID-19 onset and prone-position ventilation (days), median (IQR) & $16.0(11.0-20.0)$ & $24.0(21.0-24.0)$ & 0.042 \\
\hline Prone-position ventilation, $n$ (\%) & $19(4.6)$ & $5(1.4)$ & 0.016 \\
\hline Duration between COVID-19 onset and CRRT (days), median (IQR) & $20.5(18.8-23.0)$ & $22.0(19.0-25.0)$ & 0.682 \\
\hline CRRT, $n(\%)$ & $12(2.9)$ & $13(3.6)$ & 0.806 \\
\hline Duration between COVID-19 onset and ECMO (days), median (IQR) & $22.0(20.5-24.5)$ & $23.5(19.8-25.0)$ & $>0.99$ \\
\hline $\mathrm{ECMO}, n(\%)$ & $3(0.7)$ & $4(1.1)$ & 0.713 \\
\hline
\end{tabular}

ICU, intensive care unit; ARDS, acute respiratory distress syndrome; DIC, diffuse intravascular coagulation; AHI, acute hepatic injury; AKI, acute kidney injury; CRRT, continuous renal replacement therapy; ECMO, extracorporeal membrane oxygenation.

explored treatment effects in the following subgroups: high $(>200 \mathrm{mg}$ hydrocortisone equivalent per day) and low dose ( $\leq 200 \mathrm{mg}$ of hydrocortisone equivalent); long ( $\geq 6$ days) and short ( $<6$ days) course; early ( $\leq 3$ days of hospitalization) and late ( $>3$ days of hospitalization) initi- ation versus no corticosteroid treatment. All subgroup analyses were performed in the matched samples using 2 approaches: first, a logistic regression model that treated corticosteroid therapy as a category variable and was adjusted by PS; second, an extended Cox regression 
model that treated corticosteroids as a time-varying exposure variable and was adjusted by PS.

Data were analyzed using SPSS software (version 22.0) and R software (version 3.6.2). A $P$ value less than 0.05 was considered significant.

\section{Study approval}

This study was approved by the Medicine Institutional Review Board of Wuhan Jinyin-tan Hospital (KY-2020-03.01).

\section{Author contributions}

JL wrote this manuscript and organized tables and figures. SZ was responsible for statistical analysis and figures construction. $\mathrm{XD}$, ZL, QX, and HF were responsible for clinical data collection. JC, SH, JG, LZ, YC, WZ, HD, YL, TW, LC, and ZW were responsible for data extraction and verification. DC and JQ designed and guided this study. DA revised the raw manuscript. JL, SZ, XD, ZL, $\mathrm{QX}$, and HF contributed equally and shared first authorship.

\section{Acknowledgments}

We thank all the staff who take care of COVID-19 patients, and all the patients and their families included in the current study.

Address correspondence to: Dechang Chen or Jieming Qu, no. 197, Ruijin 2nd Road, Shanghai 200025, China. Email: chendechangsh@hotmail.com (DC) or jmqu0906@163.com (JQ). Or to: Djillali Annane, Department of Intensive Care, Hôpital Raymond Poincaré (APHP), Laboratory of Infection \& Inflammation U1173, School of Medicine Simone Veil, University Versailles Saint Quentin - University Paris Saclay, INSERM, Garches 92380, France. Email: djillali.annane@aphp.fr.
1. World Health Organization. Coronavirus disease (COVID-19) weekly epidemiological update and weekly operational update. https://www. who.int/docs/default-source/coronaviruse/ situation-reports/20200717-covid-19-sitrep-179. pdf?sfvrsn=2f1599fa_ 2 . Accessed July 17, 2020.

2. Li L, et al. Therapeutic strategies for critically ill patients with COVID-19. Ann Intensive Care. 2020;10(1):45.

3. Wu CM, et al. Risk factors associated with acute respiratory distress syndrome and death in patients with coronavirus disease 2019 pneumonia in Wuhan, China. JAMA Intern Med. 2020;180(7):934-943.

4. Zhou F, et al. Clinical course and risk factors for mortality of adult inpatients with COVID-19 in Wuhan, China: a retrospective cohort study. Lancet. 2020;395(10229):1054-1062.

5. McGonagle D, Sharif K, O'Regan A, Bridgewood C. The role of cytokines including interleukin- 6 in COVID-19 induced pneumonia and macrophage activation syndrome-like disease. Autoimmun Rev. 2020;19(6):102537.

6. Zhao Y, et al. High levels of virus-specific CD4 $\mathrm{T}$ cells predict severe pandemic influenza A virus infection. Am J Respir Crit Care Med. 2012;186(12):1292-1297.

7. Cain D, Cidlowski J. Immune regulation by glucocorticoids. Nat Rev Immunol. 2017;17(4):233-247.

8. Chen RC, et al. Treatment of severe acute respiratory syndrome with glucosteroids: the Guangzhou experience. Chest. 2006;129(6):1441-1452.

9. Sung JJ, et al. Severe acute respiratory syndrome: report of treatment and outcome after a major outbreak. Thorax. 2004;59(5):414-420.

10. Stockman LJ, Bellamy R, Garner P. SARS: systematic review of treatment effects. PLoS Med. 2006;3(9):e343.

11. Arabi YM, et al. Corticosteroid therapy for critically ill patients with Middle East respiratory syndrome. Am J Respir Crit Care Med. 2018;197(6):757-767.

12. Gibson PG, Qin L, Puah SH. COVID-19 acute respiratory distress syndrome (ARDS): clinical features differences from typical pre-COVID-19
ARDS. Med J Aust. 2020;213(2):54-56.

13. Wong CK, et al. Plasma inflammatory cytokines and chemokines in severe acute respiratory syndrome. Clin Exp Immunol. 2004;136(1):95-103.

14. Chen N, et al. Epidemiological and clinical characteristics of 99 cases of 2019 novel coronavirus pneumonia in Wuhan, China: a descriptive study. Lancet. 2020;395(10223):507-513.

15. Tsai MJ, et al. Impact of corticosteroid treatment on clinical outcomes of influenza-associated ARDS: a nationwide multicenter study. Ann Intensive Care. 2020;10(1):26.

16. Tang BM, Craig JC, Eslick GD, Seppelt I, McLean AS. Use of corticosteroids in acute lung injury and acute respiratory distress syndrome: a systematic review and meta-analysis. Crit Care Med. 2009;37(5):1594-1603.

17. Russell CD, Millar JE, Baillie JK. Clinical evidence does not support corticosteroid treatment for 2019-nCoV lung injury. Lancet. 2020;395(10223):473-475.

18. Tai DY. Pharmacologic treatment of SARS: current knowledge and recommendations. Ann Acad Med Singap. 2007;36(6):438-443.

19. Fadel R, et al. COVID-19 management task force. Early short course corticosteroids in hospitalized patients with COVID-19 [published online May 19, 2020]. Clin Infect Dis. https://doi. org/10.1093/cid/ciaa601.

20. RECOVERY Collaborative Group, et al. Dexamethasone in hospitalized patients with Covid19-preliminary report [published online May 19, 2020]. N Engl JMed. https://doi.org/10.1056/ NEJMoa2021436.

21. Yang Z, Liu J, Zhou Y, Zhao X, Zhao Q, Liu J. The effect of corticosteroid treatment on patients with coronavirus infection: a systematic review and meta-analysis. J Infect. 2020;81(1):e13-e20.

22. Lee N, et al. Effects of early corticosteroid treatment on plasma SARS-associated coronavirus RNA concentrations in adult patients. JClin Virol. 2004;31(4):304-309.

23. Buisson CB, Richard JCM, Mercat A, Thiebaut ACM, Brochard L, REVA-SRLF A/H1N1V 2009 Registry Group. Early corticosteroids in severe influenza A/H1N1 pneumonia and acute respiratory distress syndrome. Am J Respir Crit Care Med. 2011;183(9):1200-1206.

24. National Health Commission of the People's Republic of China. Diagnosis and treatment of COVID-19 guidelines (sixth version). http:// www.nhc.gov.cn/yzygj/s7653p/202002/ 8334a8326dd94d329df351d7da8aefc2/files/ b218cfeb1bc54639af227f922bf6b817.pdf. Accessed July 17, 2020.

25. Metlay JP, et al. Diagnosis and treatment of adults with community-acquired pneumonia. An official clinical practice guideline of the American Thoracic Society and Infectious Diseases Society of America. Am J Respir Crit Care Med. 2019;200(7):e45-e67.

26. ARDS Definition Task Force, et al. Acute respiratory distress syndrome: the Berlin Definition. JAMA. 2012;307(23):2526-2533.

27. Chen W, Janz DR, Shaver CM, Bernard GR, Bastarache JA, Ware LB. Clinical characteristics and outcomes are similar in ARDS diagnosed by oxygen saturation $/ \mathrm{FiO}_{2}$ ratio compared with $\mathrm{PaO}_{2} /$ $\mathrm{FiO}_{2}$ ratio. Chest. 2015;148(6):1477-1483.

28. DesPrez K, McNeil JB, Wang C, Bastarache JA, Shaver CM, Ware LB. Oxygenation saturation index predicts clinical outcomes in ARDS. Chest. 2017;152(6):1151-1158.

29. López-Fernández YM, et al. Prognostic relevance and inter-observer reliability of chest-imaging in pediatric ARDS: a pediatric acute respiratory distress incidence and epidemiology (PARDIE) study. Intensive Care Med. 2020;46(7):1382-1393.

30. Khwaja A. KDIGO clinical practice guidelines for acute kidney injury. Nephron Clin Pract. 2012;120(4):c179-c184.

31. Knaus WA, Draper EA, Wagner DP, Zimmerman JE. APACHE II: a severity of disease classification system. Crit Care Med. 1985;13(10):818-829.

32. Vincent JL, et al. The SOFA (sepsis-related organ failure assessment) score to describe organ dysfunction/failure. On behalf of the Working Group on Sepsis-Related Problems of the European Society of Intensive Care Medicine. Intensive Care Med.1996;22(7):707-710. 